\title{
Phonon-magnon resonant processes with relevance to acoustic spin pumping
}

\author{
P. A. Deymier, ${ }^{1}$ J. O. Vasseur, ${ }^{2}$ K. Runge, ${ }^{1}$ A. Manchon, ${ }^{3}$ and O. Bou-Matar ${ }^{4}$ \\ ${ }^{1}$ Department of Materials Science and Engineering, University of Arizona, Tucson, Arizona 85721, USA \\ ${ }^{2}$ Institut d'Electronique, de Micro-électronique et de Nanotechnologie, UMR CNRS 8520, Cité Scientifique, \\ 59652 Villeneuve d'Ascq Cedex, France \\ ${ }^{3}$ King Abdullah University of Science and Technology (KAUST), Physical Science and Engineering Division, \\ Thuwal 23955-6900, Saudi Arabia \\ ${ }^{4}$ International Associated Laboratory LEMAC/LICS: IEMN, UMR CNRS 8520, PRES Lille Nord de France, Ecole Centrale Lille, \\ 59652 Villeneuve d'Ascq, France
}

(Received 24 September 2014; revised manuscript received 3 December 2014; published 23 December 2014)

\begin{abstract}
The recently described phenomenon of resonant acoustic spin pumping is due to resonant coupling between an incident elastic wave and spin waves in a ferromagnetic medium. A classical one-dimensional discrete model of a ferromagnet with two forms of magnetoelastic coupling is treated to shed light on the conditions for resonance between phonons and magnons. Nonlinear phonon-magnon interactions in the case of a coupling restricted to diagonal terms in the components of the spin degrees of freedom are analyzed within the framework of the multiple timescale perturbation theory. In that case, one-phonon-two-magnon resonances are the dominant mechanism for pumping. The effect of coupling on the dispersion relations depends on the square of the amplitude of the phonon and magnon excitations. A straightforward analysis of a linear phonon-magnon interaction in the case of a magnetoelastic coupling restricted to off-diagonal terms in the components of the spins shows a one-phonon to one-magnon resonance as the pumping mechanism. The resonant dispersion relations are independent of the amplitude of the waves. In both cases, when an elastic wave with a fixed frequency is used to stimulate magnons, application of an external magnetic field can be used to approach resonant conditions. Both resonance conditions exhibit the same type of dependency on the strength of an applied magnetic field.
\end{abstract}

DOI: 10.1103/PhysRevB.90.224421

PACS number(s): 43.20.+g, 63.20.kk, 75.30.Ds

\section{INTRODUCTION}

The production of spin polarized currents is of tremendous importance for the transport of information in the form of the spin instead of the charge of electrons. The process of generation of spin currents is an essential component in the development of spintronics. To date, several approaches have been investigated to generate spin currents. The first one utilizes nonlocal spin injection [1,2]: spin current is created by electrons flowing in a ferromagnet and diffuses in an adjacent normal metal. A second set of methods generates pure spin currents from spin pumping. A precessing magnetization (e.g., driven into ferromagnetic resonance by an external magnetic field) injects a nonequilibrium spin current into an adjacent normal metal via spin angular momentum transfer [3-5]. Another popular method that currently fosters intense efforts is the spin Hall effect (SHE), where unpolarized electrons flowing in a normal metal are asymmetrically scattered via spin-orbit coupled impurities, resulting in the generation of a transverse pure spin current [6,7]. All the above methods seek to generate pure spin currents from electrical voltages and electron flows. In fact, recent developments in the field of spintronics have demonstrated that heat (i.e., acoustic phonons) could be used to pump spin currents from (insulating or metallic) ferromagnets. The exploration of the spin Seebeck effect (SSE) [8]-i.e., the generation of spin currents using heat-has revealed that chargeless spin currents can be efficiently carried by magnons in magnetic insulators such as yttrium iron garnet (YIG) [9]. Combining elastic waves with magnons in magnetic insulators offers opportunities for the development of chargeless information control since no electrical voltages or charge current flows are needed [10]. Elastic waves have also been used recently to coherently excite the magnetization of ferromagnets, induce ultrafast demagnetization, or reorient the magnetization direction $[11,12]$.

In this context, the role of phonon-magnon coupling is particularly inspiring. Indeed, it has recently been shown that the resonant absorption of elastic waves by a ferromagnet can also drive a spin current [13]. This approach has been termed acoustic spin pumping (ASP) [14]. Acoustic spin pumping is a resonant equivalent of the SSE. The fundamental mechanism underlying ASP results from the coupling between an elastic wave (phonons) and spin waves (magnons) through the magnetoelastic effect [15]. Of other significant relevance is the role played by phonon-magnon interactions and the magnetoelastic effect in the phenomenon of phonon drag enhancement of SSE [16] or the development of the field of spin caloritronics $[17,18]$.

Several theories of the magnetoelastic effect have relied on (a) approximating the ferromagnet as a continuum and (b) applying the quasistatic approximation whereby magnons are at equilibrium with respect to the slower phonons [19-21]. More recently, methods based on molecular and spin-dynamics have been employed to investigate numerically phonon-magnon interactions. These numerical methods simultaneously solve the equations of motion for atoms and spins [22-24]. Some of these studies have shown softening and damping of magnon modes due to lattice vibrations and the existence of coupled magnon-phonon modes with identical frequencies [22]. In light of the importance of magnon-phonon interactions for a wide range of emerging scientific and technical fields, we have developed an analytical approach to shed light on resonant magnon-phonon processes in models of ferromagnetic media. 
We focus on a one-dimensional model composed of atomic sites that support lattice vibrations and spin precession. We solve the coupled magnetoelastic equations of motion for the spin degrees of freedom and the atomic displacement using analytical mathematical methods. We have considered two cases, namely a case with a nonlinear magnetoelastic coupling and another one with linear coupling. While analyzing the linear system is straightforward, the nonlinear system requires the use of an approach based on the multiple time scale perturbation theory [25]. This approach enables us to obtain analytical solutions for the wave representation of the nonlinearly interacting phonons and magnons. We derive the conditions for resonance between a phonon and a magnon with an identical wave number in both cases. Phonon-magnon resonance leads to shifts in the dispersion bands of both excitations. We also demonstrate that the condition for resonance is modified by the application of an external magnetic field. The strength of the applied field can be used to tune the ferromagnetic medium to achieve resonance.

This paper is organized as follows. In Sec. II, we introduce the Hamiltonian for a discrete one-dimensional atomic model with two forms of magnetoelastic coupling. One form leads to a nonlinear coupling between phonons and magnons. The nonlinear phonon-magnon interactions arise from a coupling contribution to the Hamiltonian restricted to diagonal terms in the components of the spin degrees of freedom. A linear phonon-magnon interaction is obtained in the case of a coupling restricted to off-diagonal terms in the components of the spins. The coupled equations of motion for the spin degrees of freedom and the lattice vibrations in both cases are derived in that same section. The multiple timescale perturbation method is applied to analyzing the nonlinear equations of motion in the Appendix. In the Appendix, we derive the sets of equations of motion to zeroth, first, and second order in the phonon-magnon coupling constant. We also find analytical solutions to these sets of nonlinear equations. We show that the mechanism for energy pumping between phonons and magnons is a resonance involving one phonon and two magnons. This leads to frequency shifts and modifications of the dispersion relations that are proportional to the square of the amplitude of the elastic and magnetic waves as discussed in Sec. III. The effect of the magnetic and elastic physical characteristics of the systems on the dispersion bands are also discussed in terms of their softening and hardening in Sec. III. We also consider the effect of an externally applied magnetic field on the phonon-magnon resonance condition. Even though an external field adds only a linear term in the equations of motion of the spin degrees of freedom, we show that the condition for phonon-magnon resonance is sensitive to the strength of the external field. In Sec. IV, we solve the set of equations of motions for the linearly coupled phonons and magnons. We find in that case that a single phonon interacts with a single magnon. The resonance that drives energy pumping between phonons and magnons is involving one phonon and one magnon. As expected, it occurs when both excitations have the same phase velocity. Modifications to the dispersion relations due to the resonance are here independent of the amplitude of the waves. The effect of an external magnetic field on the one-phonon-one-magnon resonance condition leads to observations similar to those made in the more complex nonlinear case. Finally, in Sec. V, we summarize our findings and draw conclusions concerning the observed phonon-magnon resonances in the context of acoustic spin pumping.

\section{MODEL}

\section{A. Hamiltonian}

We consider a one-dimensional discrete model of a medium that can support coupled spin waves and elastic waves (see Fig. 1). This is used as a model of a ferromagnetic material with magnetoelastic coupling. This model is constituted of a chain of atoms. Each atomic site $n$ is characterized by a spin $\vec{S}_{n}$ and a displacement $u_{n}$. We assume that the model is limited to first nearest neighbor interactions. The Hamiltonian for this system is taking the form

$$
\begin{aligned}
H= & -2 \sum_{n} \sum_{i, j=x, y, z} J_{n, n+1}^{i, j}\left(u_{n+1}, u_{n}\right) S_{n}^{i} S_{n+1}^{j} \\
& +\frac{1}{2} \sum_{n} \beta\left(u_{n+1}-u_{n}\right)^{2} .
\end{aligned}
$$

In this equation, the atoms are interacting elastically via a harmonic potential with a uniform, constant stiffness $\beta$. The remaining part of the Hamiltonian is that of a Heisenberg model where the nearest neighbor spin exchange coupling constant $J_{n, n+1}^{i, j}\left(u_{n+1}, u_{n}\right)$ depends on the displacement of the atoms. The superscripts $i$ and $j$ run over all directions $x, y$, and $z$.

We consider two cases for the magnetoelastic coupling, namely case I [Eq. (2a)] and case II [Eq. (2b)]

$$
\begin{aligned}
J_{n, n+1}^{i, j}\left(u_{n+1}, u_{n}\right) & =J_{n, n+1}\left(u_{n+1}, u_{n}\right) \\
& =J \delta_{i j}-K\left(u_{n+1}-u_{n}\right) \delta_{i j}, \\
J_{n, n+1}^{i, j}\left(u_{n+1}, u_{n}\right) & =J \delta_{i j}-K_{T}\left(u_{n+1}-u_{n}\right)\left(1-\delta_{i j}\right) .
\end{aligned}
$$

In the first case, the coupling between the displacement and the spins is limited to terms in the Hamiltonian of the form $S_{n}^{i} S_{n+1}^{i}$. In case I, the magnetoelastic coupling is restricted to diagonal terms in the components of the spin degrees of freedom to the same coordinates. Case II corresponds to interactions limited to cross terms with respect to components of the spin degrees of freedom. The magnetoelastic coupling term in the Hamiltonian involves only terms of the form $S_{n}^{i} S_{n+1}^{j \neq i}$. Here, $J, K$, and $K_{T}$ are positive constants. The minus sign in Eqs. (2a) and (2b) indicates that the coupling between adjacent spin weakens as the separation distance increases.

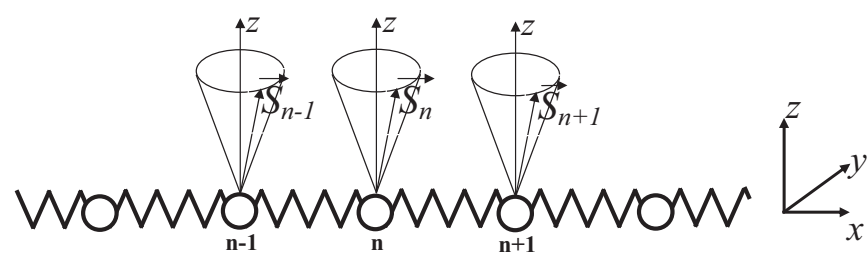

FIG. 1. Schematic illustration of the one-dimensional discrete atomic model supporting coupled spin waves and elastic waves. The $x$ axis is defined along the chain of atoms. 
The Hamiltonian for case I takes the specific form

$H=-2 \sum_{n} J_{n, n+1}\left(u_{n}, u_{n+1}\right) \vec{S}_{n} \cdot \vec{S}_{n+1}+\frac{1}{2} \sum_{n} \beta\left(u_{n+1}-u_{n}\right)^{2}$.

This Hamiltonian, then, simplifies to

$$
\begin{aligned}
H= & -2 J \sum_{n} \vec{S}_{n} \cdot \vec{S}_{n+1}+2 K \sum_{n}\left(u_{n+1}-u_{n}\right) \vec{S}_{n} \cdot \vec{S}_{n+1} \\
& +\frac{1}{2} \sum_{n} \beta\left(u_{n+1}-u_{n}\right)^{2} .
\end{aligned}
$$

The second term in Eq. (4) represents the magnetoelastic interactions between spin and displacement. As discussed previously, magnetoelastic coupling is composed only of terms diagonal in the components of the spin degrees of freedom; that is, these interactions involve only pairs of components: $x x, y y$, and $z z$ by virtue of the dot product.

In case II considered here, the Hamiltonian is written as

$$
\begin{aligned}
H= & -2 J \sum_{n} \vec{S}_{n} \cdot \vec{S}_{n+1}+2 K_{T} \sum_{n}\left(u_{n+1}-u_{n}\right) \\
& \times\left(S_{n}^{x} S_{n+1}^{y}+S_{n}^{y} S_{n+1}^{x}+S_{n}^{z} S_{n+1}^{x}+S_{n}^{x} S_{n+1}^{z}\right. \\
& \left.+S_{n}^{z} S_{n+1}^{y}+S_{n}^{y} S_{n+1}^{z}\right)+\frac{1}{2} \sum_{n} \beta\left(u_{n+1}-u_{n}\right)^{2} .
\end{aligned}
$$

The second term in Eq. (5) involves products of different components of the spin degrees of freedom; that is, these interactions involve only pairs of mixed components of the type: $x y, y z$, and $x z$.

To relate the Hamiltonians given by Eqs. (4) and (5) to more familiar forms, we refer to the continuum limit of the magnetoelastic energy for ferromagnetic materials (see Eq. (8) of Ref. [26]). In general, this energy involves all possible products of two components of the spins and all possible polarizations of the displacement. For a cubic material, the magnetoelastic energy reduces to two terms (see Eq. (10) of Ref. [26]). The first term corresponds to the interaction between the diagonal components of the strain and spins. This term is equivalent to the discrete form of the magnetoelastic energy of Eq. (4). The second term represents interactions between the shear components of the strain with the mixed components of the spins. This second term is isomorphic to that of Eq. (5). For a cubic material, the scalar quantity $u_{n}$ in Eq. (4) refers to a longitudinal displacement, while in Eq. (5), it corresponds to a transverse displacement (perpendicular to the chain). For cubic symmetry, the Hamiltonian of Eq. (4) is therefore modeling longitudinal waves/spin waves interactions, while the Hamiltonian of Eq. (5) is used to describe shear waves/spin waves interactions. We have separated these two contributions in two Hamiltonians for the sake of analytical simplicity and to unravel distinct characteristics of interactions between spin waves and transverse and longitudinal phonons. We would like to note that for noncubic symmetries, the Hamiltonian of Eq. (5) may also be representative of the interactions between longitudinal displacements and spin waves.

\section{B. Equations of motion for the spin degrees of freedom}

The dynamics of a spin are given by the Landau-Lifshitz equation

$$
\frac{\partial \vec{S}_{n}}{\partial t}=-\gamma \vec{S}_{n} \times \vec{h}_{n}
$$

where

$$
\vec{h}_{n}=\frac{1}{\gamma \hbar} \cdot \frac{\partial H}{\partial \vec{S}_{n}}
$$

is the effective magnetic field at site $n$. In Eq. (6), $\gamma(>0)$ and $\hbar$ stand for the atomic gyromagnetic ratio and the reduced Planck's constant, respectively. For the sake of simplicity, we neglect magnetic damping.

Using the Hamiltonian of Eq. (4) that corresponds to case I, the spin equation of motion becomes

$$
\begin{aligned}
\frac{\partial \vec{S}_{n}}{\partial t}= & 2 \frac{J}{\hbar} \vec{S}_{n} \times\left(\vec{S}_{n-1}+\vec{S}_{n+1}\right)-2 \frac{K}{\hbar}\left[\left(u_{n+1}-u_{n}\right) \vec{S}_{n}\right. \\
& \left.\times \vec{S}_{n+1}+\left(u_{n}-u_{n-1}\right) \vec{S}_{n} \times \vec{S}_{n-1}\right] .
\end{aligned}
$$

We seek a solution in the form

$$
\begin{aligned}
& \vec{S}_{n}=\vec{S}_{o}+\vec{\varepsilon}_{n}=\left(\begin{array}{c}
0 \\
0 \\
S_{z}^{0}
\end{array}\right)+\left(\begin{array}{c}
\varepsilon_{n, x} \\
\varepsilon_{n, y} \\
\varepsilon_{n, z}
\end{array}\right) \text { with } \\
& \varepsilon_{n, x}, \varepsilon_{n, y}, \varepsilon_{n, z} \ll S_{z}^{0} .
\end{aligned}
$$

Here, $\vec{S}_{o}$ is the magnetic moment of the spin and is oriented along the $z$ axis in the positive direction. Also, $\varepsilon_{n, z}$ must be a second-order term to satisfy the conservation of the norm of $\vec{S}_{n}$.

We expand terms of the form $\vec{S}_{n} \times \vec{S}_{n-1}$ up to second order in $\varepsilon$

$$
\begin{aligned}
\vec{S}_{n} \times \vec{S}_{n-1} \sim & \left(\begin{array}{c}
-S_{z}^{0}\left(\varepsilon_{n-1, y}-\varepsilon_{n, y}\right) \\
+S_{z}^{0}\left(\varepsilon_{n-1, x}-\varepsilon_{n, x}\right) \\
0
\end{array}\right) \\
& +\left(\begin{array}{c}
0 \\
0 \\
\varepsilon_{n, x} \varepsilon_{n-1, y}-\varepsilon_{n, y} \varepsilon_{n-1, x}
\end{array}\right) .
\end{aligned}
$$

With this, Eq. (6a) expressed in component form becomes

$$
\begin{aligned}
\frac{\partial \varepsilon_{n, x}}{\partial t}= & -2 \frac{J}{\hbar} S_{z}^{0}\left(\varepsilon_{n+1, y}-2 \varepsilon_{n, y}+\varepsilon_{n-1, y}\right) \\
& +2 \frac{K}{\hbar} S_{z}^{0}\left[\left(u_{n+1}-u_{n}\right)\left(\varepsilon_{n+1, y}-\varepsilon_{n, y}\right)\right. \\
& \left.+\left(u_{n}-u_{n-1}\right)\left(\varepsilon_{n-1, y}-\varepsilon_{n, y}\right)\right] \\
\frac{\partial \varepsilon_{n, y}}{\partial t}= & +2 \frac{J}{\hbar} S_{z}^{0}\left(\varepsilon_{n+1, x}-2 \varepsilon_{n, x}+\varepsilon_{n-1, x}\right) \\
& -2 \frac{K}{\hbar} S_{z}^{0}\left[\left(u_{n+1}-u_{n}\right)\left(\varepsilon_{n+1, x}-\varepsilon_{n, x}\right)\right. \\
& \left.+\left(u_{n}-u_{n-1}\right)\left(\varepsilon_{n-1, x}-\varepsilon_{n, x}\right)\right], \\
\frac{\partial \varepsilon_{n, z}}{\partial t}= & 2 \frac{J}{\hbar}\left[\varepsilon_{n, x}\left(\varepsilon_{n-1, y}+\varepsilon_{n+1, y}\right)-\varepsilon_{n, y}\left(\varepsilon_{n-1, x}+\varepsilon_{n+1, x}\right)\right] .
\end{aligned}
$$

The first term on the right-hand side of Eqs. (10a) and (10b) are the usual first-order terms that appear in the equations of 
motion of spin waves. All other terms that involve the constant $K$ are second-order terms resulting from the coupling between displacement and spin. Equation (10c) is second order and couples the directions $x$ and $y$ to direction $z$.

For case II, application of Eq. (6b) to the Hamiltonian given by Eq. (5) results in

$$
\begin{aligned}
\vec{h}_{n}= & -2 \frac{J}{\hbar}\left(\vec{S}_{n-1}+\vec{S}_{n+1}\right)+2 \frac{K_{T}}{\hbar}\left\{\left(u_{n+1}-u_{n}\right) \vec{V}_{n+1}\right. \\
& \left.+\left(u_{n}-u_{n-1}\right) \vec{V}_{n-1}\right\},
\end{aligned}
$$

where the vector $\vec{V}_{n}$ is defined as $\begin{gathered}S_{n}^{y}+S_{n}^{z} \\ \left(S_{n}^{x}+S_{n}^{z}\right. \\ S_{n}^{x}+S_{n}^{y}\end{gathered}$. Using Eq. (8), we approximate to the zeroth order this vector by the constant vector $\left(S_{z}^{0}\right)$. This vector is independent of location along the chain of atoms. Using this, we obtain the equations of motion for the $x$ and $y$ components to first order in displacement

$$
\begin{aligned}
\frac{\partial \varepsilon_{n, x}}{\partial t}= & -2 \frac{J}{\hbar} S_{z}^{0}\left(\varepsilon_{n+1, y}-2 \varepsilon_{n, y}+\varepsilon_{n-1, y}\right) \\
& +2 \frac{K_{T}}{\hbar}\left(S_{z}^{0}\right)^{2}\left(u_{n+1}-u_{n-1}\right), \\
\frac{\partial \varepsilon_{n, y}}{\partial t}= & +2 \frac{J}{\hbar} S_{z}^{0}\left(\varepsilon_{n+1, x}-2 \varepsilon_{n, x}+\varepsilon_{n-1, x}\right) \\
& -2 \frac{K_{T}}{\hbar}\left(S_{z}^{0}\right)^{2}\left(u_{n+1}-u_{n-1}\right),
\end{aligned}
$$

We do not have to consider an equation of motion for the component $\varepsilon_{n, z}$ since, in case II, all equations can be expressed to first order only.

\section{Equations of motion for the displacement}

In case $\mathrm{I}$, the force acting on an atom $n$ is obtained as

$$
\begin{aligned}
F_{n}=-\frac{\partial H}{\partial u_{n}}= & +\beta\left(u_{n+1}-u_{n}\right)-\beta\left(u_{n}-u_{n-1}\right) \\
& +2 K\left(\vec{S}_{n} \cdot \vec{S}_{n+1}-\vec{S}_{n-1} \cdot \vec{S}_{n}\right) .
\end{aligned}
$$

Assuming that each atom has the same mass $m$, their motion is described by the equation

$m \frac{\partial^{2} u_{n}}{\partial t^{2}}=\beta\left(u_{n+1}-2 u_{n}+u_{n-1}\right)+2 K\left(\vec{S}_{n} \cdot \vec{S}_{n+1}-\vec{S}_{n-1} \cdot \vec{S}_{n}\right)$.

Utilizing Eq. (8), Eq. (14) takes its final form

$$
\begin{aligned}
m \frac{\partial^{2} u_{n}}{\partial t^{2}}= & \beta\left(u_{n+1}-2 u_{n}+u_{n-1}\right)+2 K\left(S_{z}^{0}\left(\varepsilon_{n+1, z}-\varepsilon_{n-1, z}\right)\right. \\
& \left.+\varepsilon_{n, x}\left(\varepsilon_{n+1, x}-\varepsilon_{n-1, x}\right)+\varepsilon_{n, y}\left(\varepsilon_{n+1, y}-\varepsilon_{n-1, y}\right)\right) .
\end{aligned}
$$

When $K=0$, one recovers the usual equation of motion for a monoatomic one-dimensional harmonic chain. The second term on the right-hand side of Eq. (15) models the magnetoelastic coupling to the second order.

Equations (10a)-(10c) and (15) form the complete set of equations describing the coupled motion of spins and atoms in case I. In this case, the magnetoelastic coupling is nonlinear, and we will solve these equations within the context of multiple timescale perturbation theory [25]. The method and results are detailed in the Appendix.

In case II, limiting the interaction terms to the first order in the spin degrees of freedom, one gets the equation of motion for the displacement

$$
\begin{aligned}
m \frac{\partial^{2} u_{n}}{\partial t^{2}}= & \beta\left(u_{n+1}-2 u_{n}+u_{n-1}\right) \\
& +2 K_{T} S_{z}^{0}\left(\left(\varepsilon_{n+1, x}-\varepsilon_{n-1, x}\right)\right. \\
& \left.+\left(\varepsilon_{n+1, y}-\varepsilon_{n-1, y}\right)\right) .
\end{aligned}
$$

Equations (12a), (12b), and (16) are used to model the dynamics of the displacement and the spin degrees of freedom in case II. Because these equations are linear, solutions are straightforward and will be derived in Sec. IV.

\section{DISCUSSION OF RESULTS FOR CASE I}

\section{A. Dispersion with magnon-phonon interactions}

First of all, we summarize the findings of the Appendix. We have seen that the dispersion relations of the magnons and of the phonons are shifted due to their mutual interaction. Both dispersion relations are re-expressed in the form

$$
\begin{gathered}
\omega_{0}^{\prime *}(k)=\omega_{0}^{\prime}(k)-K^{2} \frac{2}{\hbar} S_{z}^{0} \alpha_{0}^{2}[P(k)]_{p v}, \\
\omega_{0}^{*}(k)=\omega_{0}(k)+K^{2} \frac{\frac{4}{\hbar} S_{z}^{0}}{m \omega_{M}^{2}} \lambda_{0}^{2}[P(k) Q(k)]_{p v} .
\end{gathered}
$$

Here, $\omega_{0}^{\prime}$ and $\omega_{0}$ are the frequencies of the magnons and phonons in absence of interactions. Also, $\omega_{0}^{\prime *}$ and $\omega_{0}{ }^{*}$ are the frequencies of magnons and phonons when they interact nonlinearly. In Eq. (18), $\omega_{M}$ stands for $\sqrt{\frac{\beta}{m}}$. The frequency shift of the phonons depends on the square of the amplitude of the magnons and vice versa. The amplitudes of the magnons and phonons are given by $\lambda_{0}$ and $\alpha_{0}$, respectively. In Eqs. (17) and (18), the corrected dispersion relations involve two functions of the wave number

$$
P(k)=\frac{\sin (2 k a)-2 \sin (k a)}{\sin ^{2}(k a)-\sin ^{2}\left(\frac{k a}{2}\right)-\rho \sin \left(\frac{k a}{2}\right)},
$$

and

$$
Q(k)=\frac{\sin (2 k a)-2 \sin (k a)}{\sin ^{2}(k a)-\sin ^{2}\left(\frac{k a}{2}\right)-\rho \sin \left(\frac{k a}{2}\right)} \times \frac{\frac{1}{2}(\cos (k a)-\cos (2 k a)) \sin (k a)+\rho \sin \left(\frac{k a}{2}\right)(\sin (2 k a)-\sin (k a))}{\sin ^{2}\left(\frac{k a}{2}\right)},
$$

with the dimensionless quantity $\rho$ defined as $\rho=\frac{2 \sqrt{\beta / m}}{\frac{8 J}{\hbar} S_{z}^{0}}$. The sign of these functions determines whether the frequencies of the nonlinear coupled system are softened or hardened compared to the dispersion of the noninteracting phonons 

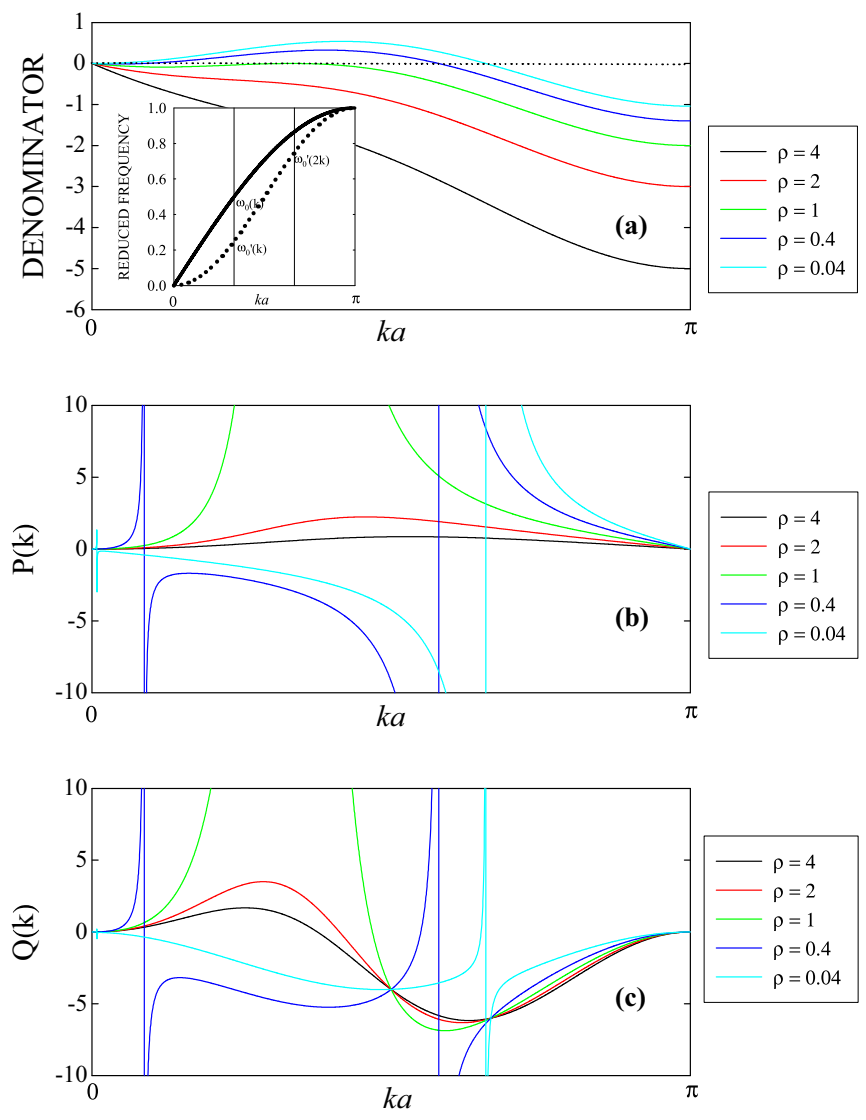

FIG. 2. (Color online) (a) Denominator of functions $P$ and $Q$ versus reduced wave number $k a$ for different values of the parameter $\rho$. The inset represents the magnon (dotted line) and the phonon (solid line) dispersion curves at $\rho=1$. The condition for resonance $\omega_{0}^{\prime}(2 k)=\left(\omega_{0}(k)+\omega_{0}^{\prime}(k)\right)$ is highlighted with vertical lines. (b) $P$ and (c) $Q$ as functions of $k a$ and $\rho$.

and magnons. In Fig. 2, we plot these functions along with the denominator of these very same functions. We vary $k a$ in the positive region of the Brillouin zone (BZ): $[0, \pi]$. The parameter $\rho=\frac{2 \sqrt{\beta / m}}{\frac{8 J}{\hbar} S S_{z}^{0}}$ measures the relative value of the cutoff frequencies of the magnons and the phonons in the chain of atoms. We investigate the cases: $\rho=4,2,1,0.4,0.04$.

Figure 2(a) illustrates the condition for phonon-magnon resonance as a function of the reduced wave number $k a$ and ratio of cutoff frequencies $\rho$. When $\rho>1$, at a given $k a$, the frequency of a phonon is always larger than that of a magnon. The denominator of the functions $P$ and $Q$ is always nonzero and negative. $P$ is positive over the entire $\mathrm{BZ}$, leading to a softening of the magnon frequency [see Eq. (17)]. Also, $Q$ is positive for small $k a$ and changes sign beyond a threshold wave number. Phonon-magnon coupling hardens the phonons at low $k a$ and softens them beyond the threshold. At $\rho=1$, a transition occurs. This corresponds to phonon and magnon bands with the same cutoff frequency. The denominator takes on a value approaching 0 at a nonvanishing wave number $(k a=1.047)$. A near resonance condition is reached when a phonon with frequency $\omega_{0}(k a=1.047)$ and a magnon with frequency $\omega_{0}^{\prime}(k a=1.047)$ interact to create a magnon of frequency $\omega_{0}^{\prime}(k a=2.094)=\omega_{0}(k a=1.047)+\omega_{0}^{\prime}(k a=$
TABLE I. Values of the parameter $\rho=\frac{2 \sqrt{\beta / m}}{\frac{8 J}{\hbar} S_{z}^{0}}=\frac{1}{4} \frac{T_{D}}{T_{C}}$ for some representative ferromagnetic materials.

\begin{tabular}{lccc}
\hline \hline Material & $T_{D}(\mathrm{~K})$ & $T_{C}(\mathrm{~K})$ & $\rho$ \\
\hline YIG & 600 & 550 & 0.2727 \\
$\mathrm{Ni}$ & 450 & 627 & 0.1794 \\
$\mathrm{Fe}$ & 470 & 1043 & 0.1126 \\
$\mathrm{Co}$ & 385 & 1388 & 0.0693 \\
\hline \hline
\end{tabular}

1.047). This resonance condition is illustrated in the inset of Fig. 2(a). The function $P$ remains positive but exhibits a large maximum near $k a=1.047$. The function $Q$ also shows a positive maximum near the resonance condition but changes sign with increasing wave number. The frequencies of the interacting magnons are softened over the complete BZ [Eq. (17)]. The frequency of the phonons increases at small wave number and decreases at larger ones in comparison to the frequency of the noninteracting phonons [Eq. (18)]. As $\rho$ decreases below the value of 1 , the denominator possesses two zeros, one at low $k a$ and one at large $k a$. These correspond to two resonance conditions. The low $k a$ resonance evolves towards a longer wavelength as $\rho$ decreases. The high $k a$ resonance moves towards the upper edge of the BZ. The functions $P$ and $Q$ change sign several times over the complete $\mathrm{BZ}$. These are the conditions that may be representative of most common ferromagnets, as will be discussed now.

The parameter $\rho$ can be related phenomenologically to the Curie temperature $T_{C}$ and the Debye temperature $T_{D}$. Ferromagnetic order breaks down at nonzero temperature for a one-dimensional Ising model with nearest neighbor interactions. However, a one-dimensional Ising model in the presence of a small external field exhibits ferromagnetism at finite temperature. At high temperature, the magnetic susceptibility follows a Curie-Weiss form with a Curie temperature defined as $T_{C}=\frac{2 J}{k_{B}}$ where $k_{B}$ is Boltzmann's constant [27]. In the preceding expression, the factor 2 relates the number of nearest neighbors of a given spin site. This is the form of the Curie temperature that would be obtained erroneously when the one-dimensional system is treated with a mean field approach. In spite of the inability of the mean field model to capture the physics of the one-dimensional system, we employ this temperature as a phenomenological measure of the thermal equivalent of the energy of our magnetic excitations. Defining the Debye frequency $v_{D}=\frac{1}{2 \pi} 2 \sqrt{\frac{\beta}{m}}$ enables us to introduce the Debye temperature $T_{D}=\frac{h v_{D}}{k_{B}}$. With these, and taking $S_{z}^{0}=1$, the parameter $\rho$ can be expressed in terms of Curie and Debye temperatures as $\rho=\frac{1}{4} \frac{T_{D}}{T_{C}}$. In Table I, we report some values of the parameter $\rho$ for some representative ferromagnetic materials.

We note that these values are less than 1 and fall within the lower range of the cases we discuss in Fig. 2. Again, we have used a one-dimensional-system mean field formula for the Curie temperature. Should we have considered threedimensional structures, $T_{C}=\frac{z J}{k_{B}}$ where $z$ is the number of nearest neighbors. This number would range from 6 to 8 to 12 for simple cubic, body-centered-cubic, and face-centeredcubic structures, effectively increasing the value of $\rho$ by factors 
of 3,4 , or 6 , respectively. Even with such multiplicative factors, the parameter $\rho$ would remain less than 1 for common ferromagnetic media.

\section{B. Effect of an external magnetic field}

We can also consider the nonlinear magnon-phonon interactions in the presence of an external magnetic field $\vec{b}$ oriented along the $\mathrm{z}$ direction. In this case, Eq. (6b) becomes $\vec{h}_{n}=\vec{b}+\frac{1}{\gamma \hbar} \cdot \frac{\partial H}{\partial \vec{S}_{n}}$. This adds the linear terms $\gamma\left|b_{z}\right| \varepsilon_{n, y}$ and $-\gamma\left|b_{z}\right| \varepsilon_{n, x}$ to the right-hand sides of Eqs. (10a) and (10b), respectively. Here, $\left|b_{z}\right|$ is the intensity of the external field. These linear terms do not affect the form of the results concerning the frequency shifts in the dispersion relations obtained with the multiple timescale perturbation approach used previously. To account for these linear terms, one simply needs to replace the magnon dispersion relation given by Eq. (A7) by $\omega_{0}^{\prime}=\frac{8 J}{\hbar} S_{z}^{0} \sin ^{2}\left(\frac{k a}{2}\right)+\gamma\left|b_{z}\right|$. The external field shifts the noninteracting magnon dispersion curve by a constant proportional to the $z$ th component of the field.

By employing this zeroth-order magnon dispersion relation, we can rewrite the function $d(k)$, defined by Eq. (A35), in the form

$$
d(k)=\omega_{0}^{\prime}(2 k)-\gamma\left|b_{z}\right|-\left(\omega_{0}(k)+\omega_{0}^{\prime}(k)\right)+i \psi .
$$

In the absence of damping and a magnetic field, this expression gives a condition for the divergence (resonance) of the function $f(k)$. This divergence corresponds to a process involving a phonon with wave number $k$ and frequency $\omega_{0}(k)$ interacting with a magnon with the same wave number but a frequency $\omega_{0}^{\prime}(k)$ to produce a magnon with wave number $2 k$ and frequency $\omega_{0}^{\prime}(2 k)=\left(\omega_{0}(k)+\omega_{0}^{\prime}(k)\right)$. This corresponds to a single-phonon-two-magnon resonant process [28]. Keeping this picture of phonon-magnon interactions as a basis for our discussion, the application of an external magnetic field effectively modifies the phonon resonant frequency to a value given by $\omega_{0}(k)+\gamma\left|b_{z}\right|$. Therefore, using a fixed frequency phonon to excite magnons, one can apply a variable external field to reach resonance. On the other hand, one can apply a fixed magnetic field and change the phonon frequency to attain the resonance condition. This is illustrated by rewriting Eq. (19) in the form of the following resonance condition

$$
\Delta \omega_{0}^{\prime}=\omega_{0}^{\prime}(2 k)-\omega_{0}^{\prime}(k)=\omega_{0}(k)+\gamma\left|b_{z}\right| .
$$

Note that, in this relation, the left-hand term is independent of an applied magnetic field. Consider now that the phonon frequency $\omega_{0}(k)$ is fixed. That is, we excite the system with a device such as an interdigitated transducer that emits elastic waves over a narrow band (i.e., produces monochromatic elastic waves with a nearly fixed frequency and wave number). In that case, the quantity $\Delta \omega_{0}^{\prime}$ is also a constant. Let us assume that the system is subjected to an applied field $b_{z}^{0}$ such that the resonance condition is not met; that is, $\Delta \omega_{0}^{\prime}(k) \neq$ $\omega_{0}(k)+\gamma\left|b_{z}^{0}\right|$. It is therefore possible to detune positively or negatively the magnitude of the external field $\left|b_{z}^{0}\right| \pm \Delta b$ to approach the condition for resonance given by Eq. (20). In Ref. [13], Weiler et al. have studied the attenuation of a surface acoustic wave (SAW) launched in a ferromagnetic cobalt film as a function of the magnitude of an external magnetic field applied in some specific direction. The frequency of the SAW was constant and determined by the interdigitated transducer that emitted it. These authors observed a resonant absorption of the SAW at a distinct value of the magnetic field strength in accordance with our one-phonon-two-magnon resonant process prediction. In Ref. [15], a similar study was conducted for a nickel film. The strength of the magnetic field necessary to achieve resonance was shown to increase with increasing SAW frequency. This could be explained in the context of our one-phonon-two-magnon resonant process. Indeed, in the long wavelength limit, $\Delta \omega_{0}^{\prime}(k)$ increases rapidly with increasing wave number owing to the quadratic form of the magnon dispersion relation. In that same limit, the frequency of the phonons increases linearly with the wave number. One therefore needs to increase the value of $\gamma\left|b_{z}\right|$ (i.e., the strength of the external field) as one increases the wave number (i.e., phonon frequency) to maintain the condition for resonance.

\section{ANALYSIS OF CASE II}

In Sec. III, we have addressed the coupling between phonons and magnons in case I, whereby the interactions between these excitations are nonlinear. In this section, we derive the solutions of the equations of motion in Eqs. (12a), (12b), and (16), which correspond to a linear coupling between phonons and magnons. The linear interactions arose from the fact that we limited the magnetoelastic coupling to cross terms in the components of the spin in the system's Hamiltonian. In this case, we assume that the spin degrees of freedom and the displacement take a plane wave form

$$
\begin{aligned}
\varepsilon_{n, x} & =\varepsilon_{o, x} e^{i k n a} e^{i \omega t}, \\
\varepsilon_{n, y} & =\varepsilon_{o, y} e^{i k n a} e^{i \omega t}, \\
u_{n} & =A_{o} e^{i k n a} e^{i \omega t} .
\end{aligned}
$$

Inserting these solutions into the equations of motion leads to a simple eigenvalue problem. The frequency of the linearly coupled phonon and magnon is subsequently obtained as

$$
\omega^{2}=\frac{\omega_{0}^{\prime 2}+\omega_{0}^{2}}{2} \pm \frac{1}{2} \sqrt{\left(\omega_{0}^{\prime 2}-\omega_{0}^{2}\right)^{2}+\frac{\frac{8}{\hbar} S_{z}^{0} B^{2}}{m} \omega_{0}^{\prime}},
$$

where $B=4 K_{T} S_{z}^{0} \sin (k a)$.

In Eq. (22), we have used the expressions for the eigenvalues of the phonons and magnons in the absence of interactions given by Eqs. (A7) and (A10) (see Appendix). If an external magnetic field is applied, the frequency $\omega_{0}^{\prime}$ is simply augmented by the constant term $\gamma\left|b_{z}^{0}\right|$. Equation (22) indicates that a resonance between a single magnon and a single phonon will occur when $\omega_{0}^{\prime}(k)=\omega_{0}(k)$; that is, when the elastic wave and the magnetic wave have identical phase velocities. This resonance is more easily seen when performing a Taylor expansion of Eq. (22) to the second order in $K_{T}$

$$
\begin{aligned}
\omega^{2}= & \frac{\omega_{0}^{\prime 2}+\omega_{0}^{2}}{2} \pm \frac{1}{2} \operatorname{sgn}\left\{\left(\omega_{0}^{\prime 2}-\omega_{0}^{2}\right)\right\}\left(\omega_{0}^{\prime 2}-\omega_{0}^{2}\right) \\
& \pm \operatorname{sgn}\left\{\left(\omega_{0}^{\prime 2}-\omega_{0}^{2}\right)\right\} \frac{\frac{2}{\hbar} S_{z}^{0} B^{2}}{m} \frac{\omega_{0}^{\prime}}{\left(\omega_{0}^{\prime 2}-\omega_{0}^{2}\right)} .
\end{aligned}
$$


Depending on the sign of $\omega_{0}^{\prime 2}-\omega_{0}^{2}$ (i.e., $\operatorname{sgn}\left\{\left(\omega_{0}^{\prime 2}-\omega_{0}^{2}\right)\right\}$ ), the first two terms give $\omega_{0}^{\prime 2}$ and $\omega_{0}^{2}$. The third terms represents the effect of the resonance. The effect of magnetoelastic coupling on the dispersion relations of the phonons and magnons in case II is independent of the amplitude of the waves.

We can also consider the application of an external magnetic field. In that case, an external magnetic field can be applied to the ferromagnetic medium to tune the dispersion curve of the magnons with the aim of achieving resonance under the condition of a stimulating acoustic wave with a fixed frequency. The observations concerning the dependence of strength of the applied magnetic field on the frequency of the acoustic wave made in Sec. III for the single-phonontwo-magnon resonance also transpose to the case of a singlephonon-single-magnon resonance.

\section{CONCLUSIONS}

We considered the effect of coupling between elastic waves and magnetic waves in a one-dimensional discrete model system of a ferromagnetic medium. Two cases are addressed. The first case corresponds to a system where the magnons and phonons interact nonlinearly. The nonlinear interaction is due to a displacement-dependent spin exchange coupling constant that involves only diagonal terms in the components of the spin, i.e., to the interaction between longitudinal components of the strain and spins in a cubic material. The second case leads to linear interactions resulting from a displacement-dependent spin exchange coupling constant that involves off-diagonal terms in the components of the spin, i.e., to the interaction between shear components of the strain and spins in a cubic material. We have developed an approach based on the multiple timescale perturbation method to calculate analytically the effect of the nonlinear magnetoelastic coupling on the dispersion relation of magnons and phonons. For the sake of mathematical tractability, we have limited our paper to the interaction between magnons and phonons with the same wave number. We have determined the conditions that lead to magnon-phonon resonance. In the absence of an external magnetic field, the resonance for the first case is associated with a single-phonon-two-magnon process. Such one-phonon-two-magnon processes are characteristic of the interaction between longitudinal phonons with magnons in nearest neighbor Heisenberg models where the exchange coupling constant is modulated with respect to the lattice displacements [29]. Our two-magnon-one-phonon resonance leads to a frequency shift of the magnon and phonon bands that depends on the magnitude of the elastic and magnetic waves. The frequency shift also depends on the relative magnitude of the cutoff frequencies of the two excitations. For the second case (i.e., linear coupling), the resonance includes only one phonon and one magnon. The effect of magnetoelastic coupling results in modifications to the dispersion relations that do not depend on the magnitude of the waves. We have also investigated the effect of an external field on that linear resonant condition. The behavior of the linear system with respect to the strength of the magnetic field is similar to that of the nonlinear system. We have demonstrated that, similarly to observations reported in the literature concerning the phenomena of resonant spin pumping with coherent elastic waves $[13,15]$, variation of the strength of an externally applied magnetic field at a fixed frequency of the elastic wave can be used to tune the system toward resonance. In addition, the strength of the external field at resonance increases with increasing acoustic wave frequency.

In Ref. [24], molecular dynamics simulations of coupled lattice and spin dynamics in body-centered-cubic iron have shown a softening of the magnons for all the wave vectors studied. In contrast, for iron (see Table I), we have observed softening of the spin waves for long and short wavelength waves but a hardening for intermediate wavelengths [Fig. 2(b)]. The computational studies also conjectured the existence of a resonance between longitudinal lattice vibrations and spin waves of the same frequency and wave number. We have shown that such a resonant condition arises in the case of linear interaction between phonons and magnons (case II). However, in this case, for cubic symmetries, the phonon polarization is transverse and not longitudinal. We noted in Sec. II that, for noncubic materials, such a coupling could arise with longitudinal waves. Such a situation may occur in molecular dynamics simulations through the instantaneous breaking of the symmetry of the cubic model. However, direct comparison with numerical simulations of ferromagnetic materials with interactions between spin/lattice vibrations [22-24] is clouded by the fact that computational results arise from the collective effects of multiple phonon-magnon scattering processes with differing wave numbers. Our analytical solutions are focused on the interactions between elastic and spin waves with specific wave numbers. Nevertheless, by providing analytical solutions for the spin degrees of freedom and the lattice dynamics in model ferromagnetic atomic systems, our paper enables a deeper insight into the complex interplay between magnons and phonons interacting via the magnetoelastic effect as a mechanism for spin pumping. This insight offers perspective in the design of ferromagnetic media that may optimize the energy conversion between phonons and magnons. In particular, one may be able to engineer the phonon and magnon band structures of the ferromagnetic medium to control the conditions of resonance. We have in mind composite structures composed of ferromagnetic and normal materials that can be tailored to exhibit band gaps in their magnon band structures [30]. Similarly, these types of composites may also act as phononic crystals that can simultaneously exhibit band gaps in their phonon band structure [31]. Wave localization resulting from these anomalous band structures may subsequently be employed to enhance magnetoelastic interactions and therefore the efficiency of energy conversion between phonon and magnons in acoustic spin pumping devices.

\section{APPENDIX}

\section{Multiple timescale perturbation method applied to case I}

We apply the multiple timescale perturbation method [25] to the nonlinear equations of motion of case I, given by Eq. (15). We assume that the constant $K$ that couples the spins and the displacement is small. Subsequently, we expand the spin degrees of freedom and the displacement in a power series of 
$K$ up to the second order

$$
\begin{aligned}
\varepsilon_{n, i} & =\varepsilon_{n, i}^{(0)}+K \varepsilon_{n, i}^{(1)}+K^{2} \varepsilon_{n, i}^{(2)}, \quad i=x, y, z, \\
u_{n} & =u_{n}^{(0)}+K u_{n}^{(1)}+K^{2} u_{n}^{(2)} .
\end{aligned}
$$

The quantities $\varepsilon_{n, i}^{(0)}, \varepsilon_{n, i}^{(1)}, \varepsilon_{n, i}^{(2)}, u_{n}^{(0)}, u_{n}^{(1)}, u_{n}^{(2)}$ are functions of three time-related variables defined as $\tau_{0}=t, \tau_{1}=K t$, and $\tau_{2}=K^{2} t[25]$.

The first- and second-order time derivatives on the lefthand side of Eqs. (10a)-(10c) and (15) are then rewritten as

$$
\begin{aligned}
\frac{\partial^{2} u_{n}}{\partial t^{2}}= & \frac{\partial^{2} u_{n}^{(0)}}{\partial \tau_{0}^{2}}+K\left(\frac{\partial^{2} u_{n}^{(1)}}{\partial \tau_{0}^{2}}+2 \frac{\partial^{2} u_{n}^{(0)}}{\partial \tau_{0} \partial \tau_{1}}\right) \\
& +K^{2}\left(\frac{\partial^{2} u_{n}^{(1)}}{\partial \tau_{0}^{2}}+2 \frac{\partial^{2} u_{n}^{(1)}}{\partial \tau_{0} \partial \tau_{1}}+2 \frac{\partial^{2} u_{n}^{(0)}}{\partial \tau_{0} \partial \tau_{2}}+\frac{\partial^{2} u_{n}^{(0)}}{\partial \tau_{1}^{2}}\right),
\end{aligned}
$$

$$
\begin{aligned}
\frac{\partial \varepsilon}{\partial t}= & \frac{\partial \varepsilon^{(0)}}{\partial \tau_{0}}+K\left(\frac{\partial \varepsilon^{(1)}}{\partial \tau_{0}}+\frac{\partial \varepsilon^{(0)}}{\partial \tau_{1}}\right) \\
& +K^{2}\left(\frac{\partial \varepsilon^{(2)}}{\partial \tau_{0}}+\frac{\partial \varepsilon^{(1)}}{\partial \tau_{1}}+\frac{\partial \varepsilon^{(0)}}{\partial \tau_{2}}\right),
\end{aligned}
$$

We insert Eqs. (A2a), (A2b), and (A1a), (A1b) into Eqs. (10) and (15) and separate the terms that are independent of $K$ (zeroth-order terms in $K$ ), from the first-order terms in $K$, from the terms in $K^{2}$. This operation leads to three sets of four equations.

The equations of motion to the zeroth order in $K$ are

$$
\begin{aligned}
\frac{\partial \varepsilon_{n, x}^{(0)}}{\partial \tau_{0}}= & -2 \frac{J}{\hbar} S_{z}^{0}\left(\varepsilon_{n+1, y}^{(0)}-2 \varepsilon_{n, y}^{(0)}+\varepsilon_{n-1, y}^{(0)}\right), \\
\frac{\partial \varepsilon_{n, y}^{(0)}}{\partial \tau_{0}}= & +2 \frac{J}{\hbar} S_{z}^{0}\left(\varepsilon_{n+1, x}^{(0)}-2 \varepsilon_{n, x}^{(0)}+\varepsilon_{n-1, x}^{(0)}\right), \\
\frac{\partial \varepsilon_{n, z}^{(0)}}{\partial \tau_{0}}= & +2 \frac{J}{\hbar}\left(\varepsilon_{n, x}^{(0)}\left(\varepsilon_{n+1, y}^{(0)}+\varepsilon_{n-1, y}^{(0)}\right)\right. \\
& \left.-\varepsilon_{n, y}^{(0)}\left(\varepsilon_{n+1, x}^{(0)}+\varepsilon_{n-1, x}^{(0)}\right)\right) \\
\frac{\partial^{2} u_{n}^{(0)}}{\partial \tau_{0}^{2}}= & \omega_{M}^{2}\left(u_{n+1}^{(0)}-2 u_{n}^{(0)}+u_{n-1}^{(0)}\right) .
\end{aligned}
$$

We have introduced in Eq. (A3d) the characteristic frequency $\omega_{M}=\sqrt{\frac{\beta}{m}}$.

To the first order in $K$, we obtain

$$
\begin{aligned}
& \frac{\partial \varepsilon_{n, x}^{(1)}}{\partial \tau_{0}}+\frac{\partial \varepsilon_{n, x}^{(0)}}{\partial \tau_{1}}=-2 \frac{J}{\hbar} S_{z}^{0}\left(\varepsilon_{n+1, y}^{(1)}-2 \varepsilon_{n, y}^{(1)}+\varepsilon_{n-1, y}^{(1)}\right)+\frac{2}{\hbar} S_{z}^{0}\left[\left(u_{n+1}^{(0)}-u_{n}^{(0)}\right)\left(\varepsilon_{n+1, y}^{(0)}-\varepsilon_{n, y}^{(0)}\right)-\left(u_{n}^{(0)}-u_{n-1}^{(0)}\right)\left(\varepsilon_{n, y}^{(0)}-\varepsilon_{n-1, y}^{(0)}\right)\right] \\
& \frac{\partial \varepsilon_{n, y}^{(1)}}{\partial \tau_{0}}+\frac{\partial \varepsilon_{n, y}^{(0)}}{\partial \tau_{1}}=+2 \frac{J}{\hbar} S_{z}^{0}\left(\varepsilon_{n+1, x}^{(1)}-2 \varepsilon_{n, x}^{(1)}+\varepsilon_{n-1, x}^{(1)}\right)-\frac{2}{\hbar} S_{z}^{0}\left[\left(u_{n+1}^{(0)}-u_{n}^{(0)}\right)\left(\varepsilon_{n+1, x}^{(0)}-\varepsilon_{n, x}^{(0)}\right)-\left(u_{n}^{(0)}-u_{n-1}^{(0)}\right)\left(\varepsilon_{n, x}^{(0)}-\varepsilon_{n-1, x}^{(0)}\right)\right]
\end{aligned}
$$

$$
\frac{\partial \varepsilon_{n, z}^{(1)}}{\partial \tau_{0}}+\frac{\partial \varepsilon_{n, z}^{(0)}}{\partial \tau_{1}}=2 \frac{J}{\hbar}\left[\varepsilon_{n, x}^{(1)}\left(\varepsilon_{n+1, y}^{(0)}+\varepsilon_{n-1, y}^{(0)}\right)+\varepsilon_{n, x}^{(0)}\left(\varepsilon_{n+1, y}^{(1)}+\varepsilon_{n-1, y}^{(1)}\right)-\varepsilon_{n, y}^{(1)}\left(\varepsilon_{n+1, x}^{(0)}+\varepsilon_{n-1, x}^{(0)}\right)-\varepsilon_{n, y}^{(0)}\left(\varepsilon_{n+1, x}^{(1)}+\varepsilon_{n-1, x}^{(1)}\right)\right] \text {, }
$$

$$
\begin{aligned}
\frac{\partial^{2} u_{n}^{(1)}}{\partial \tau_{0}^{2}}+2 \frac{\partial^{2} u_{n}^{(0)}}{\partial \tau_{0} \partial \tau_{1}}= & \omega_{M}^{2}\left(u_{n+1}^{(1)}-2 u_{n}^{(1)}+u_{n-1}^{(1)}\right)+\frac{2}{m} S_{z}^{0}\left(\varepsilon_{n+1, z}^{(0)}-\varepsilon_{n-1, z}^{(0)}\right) \\
& +\frac{2}{m}\left[\varepsilon_{n, x}^{(0)}\left(\varepsilon_{n+1, x}^{(0)}-\varepsilon_{n-1, x}^{(0)}\right)+\varepsilon_{n, y}^{(0)}\left(\varepsilon_{n+1, y}^{(0)}-\varepsilon_{n-1, y}^{(0)}\right)\right],
\end{aligned}
$$

The equations to the order of $K^{2}$ are

$$
\begin{aligned}
\frac{\partial \varepsilon_{n, x}^{(2)}}{\partial \tau_{0}}+\frac{\partial \varepsilon_{n, x}^{(1)}}{\partial \tau_{1}}+\frac{\partial \varepsilon_{n, x}^{(0)}}{\partial \tau_{2}}= & -2 \frac{J}{\hbar} S_{z}^{0}\left(\varepsilon_{n+1, y}^{(2)}-2 \varepsilon_{n, y}^{(2)}+\varepsilon_{n-1, y}^{(2)}\right)+\frac{2}{\hbar} S_{z}^{0}\left[\left(u_{n+1}^{(0)}-u_{n}^{(0)}\right)\left(\varepsilon_{n+1, y}^{(1)}-\varepsilon_{n, y}^{(1)}\right)+\left(u_{n+1}^{(1)}-u_{n}^{(1)}\right)\left(\varepsilon_{n+1, y}^{(0)}-\varepsilon_{n, y}^{(0)}\right)\right. \\
& \left.-\left(u_{n}^{(0)}-u_{n-1}^{(0)}\right)\left(\varepsilon_{n, y}^{(1)}-\varepsilon_{n-1, y}^{(1)}\right)-\left(u_{n}^{(1)}-u_{n-1}^{(1)}\right)\left(\varepsilon_{n, y}^{(0)}-\varepsilon_{n-1, y}^{(0)}\right)\right], \\
\frac{\partial \varepsilon_{n, y}^{(2)}}{\partial \tau_{0}}+\frac{\partial \varepsilon_{n, y}^{(1)}}{\partial \tau_{1}}+\frac{\partial \varepsilon_{n, y}^{(0)}}{\partial \tau_{2}}= & +2 \frac{J}{\hbar} S_{z}^{0}\left(\varepsilon_{n+1, x}^{(2)}-2 \varepsilon_{n, x}^{(2)}+\varepsilon_{n-1, x}^{(2)}\right)-\frac{2}{\hbar} S_{z}^{0}\left[\left(u_{n+1}^{(0)}-u_{n}^{(0)}\right)\left(\varepsilon_{n+1, x}^{(1)}-\varepsilon_{n, x}^{(1)}\right)\right. \\
& \left.+\left(u_{n+1}^{(1)}-u_{n}^{(1)}\right)\left(\varepsilon_{n+1, x}^{(0)}-\varepsilon_{n, x}^{(0)}\right)-\left(u_{n}^{(0)}-u_{n-1}^{(0)}\right)\left(\varepsilon_{n, x}^{(1)}-\varepsilon_{n-1, x}^{(1)}\right)-\left(u_{n}^{(1)}-u_{n-1}^{(1)}\right)\left(\varepsilon_{n, x}^{(0)}-\varepsilon_{n-1, x}^{(0)}\right)\right],
\end{aligned}
$$

$$
\begin{aligned}
\frac{\partial \varepsilon_{n, z}^{(2)}}{\partial \tau_{0}}+\frac{\partial \varepsilon_{n, z}^{(1)}}{\partial \tau_{1}}+\frac{\partial \varepsilon_{n, z}^{(0)}}{\partial \tau_{2}}= & 2 \frac{J}{\hbar}\left[\varepsilon_{n, x}^{(2)}\left(\varepsilon_{n+1, y}^{(0)}+\varepsilon_{n-1, y}^{(0)}\right)+\varepsilon_{n, x}^{(0)}\left(\varepsilon_{n+1, y}^{(2)}+\varepsilon_{n-1, y}^{(2)}\right)+\varepsilon_{n, x}^{(1)}\left(\varepsilon_{n+1, y}^{(1)}+\varepsilon_{n-1, y}^{(1)}\right)-\varepsilon_{n, y}^{(2)}\left(\varepsilon_{n+1, x}^{(0)}+\varepsilon_{n-1, x}^{(0)}\right)\right. \\
& \left.-\varepsilon_{n, y}^{(0)}\left(\varepsilon_{n+1, x}^{(2)}+\varepsilon_{n-1, x}^{(2)}\right)-\varepsilon_{n, y}^{(1)}\left(\varepsilon_{n+1, x}^{(1)}+\varepsilon_{n-1, x}^{(1)}\right)\right]
\end{aligned}
$$




$$
\begin{aligned}
\frac{\partial^{2} u_{n}^{(2)}}{\partial \tau_{0}{ }^{2}}+2 \frac{\partial^{2} u_{n}^{(1)}}{\partial \tau_{0} \partial \tau_{1}}+2 \frac{\partial^{2} u_{n}^{(0)}}{\partial \tau_{0} \partial \tau_{2}}+\frac{\partial^{2} u_{n}^{(0)}}{\partial \tau_{1}{ }^{2}}= & \omega_{M}^{2}\left(u_{n+1}^{(2)}-2 u_{n}^{(2)}+u_{n-1}^{(2)}\right)+\frac{2}{m} S_{z}^{0}\left(\varepsilon_{n+1, z}^{(1)}-\varepsilon_{n-1, z}^{(1)}\right)+\frac{2}{m}\left[\varepsilon_{n, x}^{(0)}\left(\varepsilon_{n+1, x}^{(1)}-\varepsilon_{n-1, x}^{(1)}\right)\right. \\
& \left.+\varepsilon_{n, x}^{(1)}\left(\varepsilon_{n+1, x}^{(0)}-\varepsilon_{n-1, x}^{(0)}\right)+\varepsilon_{n, y}^{(0)}\left(\varepsilon_{n+1, y}^{(1)}-\varepsilon_{n-1, y}^{(1)}\right)+\varepsilon_{n, y}^{(1)}\left(\varepsilon_{n+1, y}^{(0)}-\varepsilon_{n-1, y}^{(0)}\right)\right] . \quad
\end{aligned}
$$

\section{Solutions of the equations of motion in case $I$}

\section{a. Zeroth order in $\mathrm{K}$}

Here, we solve the set of Eqs. (A3a)-(A3d). Equations (A3a)-(A3c) and (A3d) are not coupled, so we can solve them separately. For Eqs. (A3a) and (A3b), we assume that the $x$ and $y$ components of the spin degree of freedom are expressed as

$\varepsilon_{n, x}^{(0)}\left(\tau_{0}, \tau_{1}, \tau_{2}\right)=X\left(\tau_{1}, \tau_{2}\right) e^{i k n a} e^{-i \omega_{0}^{\prime} \tau_{0}}+\bar{X}\left(\tau_{1}, \tau_{2}\right) e^{-i k n a} e^{i \omega_{0}^{\prime} \tau_{0}}$,

$\varepsilon_{n, y}^{(0)}\left(\tau_{0}, \tau_{1}, \tau_{2}\right)=Y\left(\tau_{1}, \tau_{2}\right) e^{i k n a} e^{-i \omega_{0}^{\prime} \tau_{0}}+\bar{Y}\left(\tau_{1}, \tau_{2}\right) e^{-i k n a} e^{i \omega_{0}^{\prime} \tau_{0}}$.

In Eqs. (A6a) and (A6b), $a$ stands for the spacing between two atoms in the chain. Here, $\bar{X}$ and $\bar{Y}$ are the complex conjugates of $X$ and $Y$. Inserting Eqs. (A6a) and (A6b) into Eqs. (A3a) and (A3b) yields two sets of linear equations of $X$ and $Y$ and $\bar{X}$ and $\bar{Y}$. The eigenvalues associated with these sets of equations are given by

$$
\omega_{0}^{\prime}=8 \frac{J}{\hbar} S_{z}^{0} \sin ^{2}\left(\frac{k a}{2}\right) .
$$

In the limit of small wave number $k$, one recovers the usual quadratic dispersion relation for spin waves or magnons in the long wavelength. The eigenvectors are found to be $Y=-i X$ and $\bar{Y}=i \bar{X}$. Then, the solutions in Eqs. (A6a) and (A6b) take the specific form

$$
\begin{aligned}
& \varepsilon_{n, x}^{(0)}=\varepsilon_{o}\left(\tau_{1}, \tau_{2}\right) e^{i k n a} e^{-i \omega_{0}^{\prime} \tau_{0}}+\bar{\varepsilon}_{0}\left(\tau_{1}, \tau_{2}\right) e^{-i k n a} e^{i \omega_{0}^{\prime} \tau_{0}}, \\
& \varepsilon_{n, y}^{(0)}=-i \varepsilon_{o}\left(\tau_{1}, \tau_{2}\right) e^{i k n a} e^{-i \omega_{0}^{\prime} \tau_{0}}+i \bar{\varepsilon}_{0}\left(\tau_{1}, \tau_{2}\right) e^{-i k n a} e^{i \omega_{0}^{\prime} \tau_{0}},
\end{aligned}
$$

where $\varepsilon_{o}$ is some yet unknown function, and $\bar{\varepsilon}_{0}$ its complex conjugate.

If one inserts Eqs. (A8a) and (A8b) into Eq. (A3c), the right-hand side term is analytically zero, and one finds that $\varepsilon_{n, z}^{(0)}$ is constant. It can be taken to be equal to zero.

Finally, we also chose a general solution for the zeroth order displacement given by

$$
\begin{aligned}
& u_{n}^{(0)}\left(\tau_{0}, \tau_{1}, \tau_{2}\right) \\
& \quad=A_{0}\left(\tau_{1}, \tau_{2}\right) e^{i k n a} e^{-i \omega_{0} \tau_{0}}+\bar{A}_{0}\left(\tau_{1}, \tau_{2}\right) e^{-i k n a} e^{i \omega_{0} \tau_{0}} .
\end{aligned}
$$

Inserting this expression into the zeroth-order equation of motion in Eq. (A3d) yields the well-known eigenvalues for elastic waves or phonons in a monoatomic harmonic chain

$$
\omega_{0}=2 \omega_{M} \sin \left(\frac{k a}{2}\right) \text {. }
$$

\section{b. First order in $K$}

We can now insert the zeroth-order solutions obtained in the preceding subsection into the first-order Eqs. (A4a)-(A4d). By doing this, we restrict our calculation to solutions whereby the magnons and phonons that interact with each other have the same wave number $k$. For instance, Eq. (A4d) can be rewritten as

$$
\begin{aligned}
& \frac{\partial^{2} u_{n}^{(1)}}{\partial \tau_{0}^{2}}-\left(u_{n+1}^{(1)}-2 u_{n}^{(1)}+u_{n-1}^{(1)}\right) \\
& \quad=2 i \omega_{0}\left(\frac{\partial A_{0}}{\partial \tau_{1}} e^{i k n a} e^{-i \omega_{0} \tau_{0}}+\frac{\partial \bar{A}_{0}}{\partial \tau_{1}} e^{-i k n a} e^{i \omega_{0} \tau_{0}}\right) .
\end{aligned}
$$

The solution of the homogeneous equation (left-hand side of the equation equal to zero) is of the form

$$
\begin{aligned}
& u_{n, h}^{(1)}\left(\tau_{0}, \tau_{1}, \tau_{2}\right) \\
& \quad=B_{0}\left(\tau_{1}, \tau_{2}\right) e^{i k n a} e^{-i \omega_{0} \tau_{0}}+\bar{B}_{0}\left(\tau_{1}, \tau_{2}\right) e^{-i k n a} e^{i \omega_{0} \tau_{0}} .
\end{aligned}
$$

The term in parenthesis on the right-hand side of Eq. (A11) leads to secular terms unless one sets it to zero by imposing $\frac{\partial A_{0}}{\partial \tau_{1}}=0$ and $\frac{\partial \bar{A}_{0}}{\partial \tau_{1}}=0$. This implies that the quantities $A_{0}$ and $\bar{A}_{0}$ are functions of $\tau_{2}$ only. This constrains the zeroth-order solution [Eq. (A9)] to have the form

$$
u_{n}^{(0)}\left(\tau_{0}, \tau_{2}\right)=A_{0}\left(\tau_{2}\right) e^{i k n a} e^{-i \omega_{0} \tau_{0}}+\bar{A}_{0}\left(\tau_{2}\right) e^{-i k n a} e^{i \omega_{0} \tau_{0}} .
$$

Similarly, the same constraint applies to the solution of the homogeneous equation [Eq. (A12)]

$$
u_{n, h}^{(1)}\left(\tau_{0}, \tau_{2}\right)=B_{0}\left(\tau_{2}\right) e^{i k n a} e^{-i \omega_{0} \tau_{0}}+\bar{B}_{0}\left(\tau_{2}\right) e^{-i k n a} e^{i \omega_{0} \tau_{0}} .
$$

We now solve the first-order equations of motion for the spin degrees of freedom. We begin with Eq. (A4a). We rewrite it in the form

$$
\begin{aligned}
\frac{\partial \varepsilon_{n, x}^{(1)}}{\partial \tau_{0}} & +2 \frac{J}{\hbar} S_{z}^{0}\left(\varepsilon_{n+1, y}^{(1)}-2 \varepsilon_{n, y}^{(1)}+\varepsilon_{n-1, y}^{(1)}\right) \\
= & -\frac{\partial \varepsilon_{n, x}^{(0)}}{\partial \tau_{1}}+\frac{2}{\hbar} S_{z}^{0}\left[\left(u_{n+1}^{(0)}-u_{n}^{(0)}\right)\left(\varepsilon_{n+1, y}^{(0)}-\varepsilon_{n, y}^{(0)}\right)\right. \\
& \left.-\left(u_{n}^{(0)}-u_{n-1}^{(0)}\right)\left(\varepsilon_{n, y}^{(0)}-\varepsilon_{n-1, y}^{(0)}\right)\right] .
\end{aligned}
$$

The solution of the homogeneous equation is given by the expression

$$
\varepsilon_{n, x, h}^{(1)}=\eta_{0}\left(\tau_{1}, \tau_{2}\right) e^{i k n a} e^{-i \omega_{0}^{\prime} \tau_{0}}+\bar{\eta}_{0}\left(\tau_{1}, \tau_{2}\right) e^{-i k n a} e^{i \omega_{0}^{\prime} \tau_{0}} .
$$

Inserting Eq. (A8a) into the first term of the right-hand side of Eq. (A15) will lead to secular terms unless one imposes the constraints $\varepsilon_{o}\left(\tau_{1}, \tau_{2}\right)=\varepsilon_{o}\left(\tau_{2}\right)$ and $\bar{\varepsilon}_{0}\left(\tau_{1}, \tau_{2}\right)=\bar{\varepsilon}_{0}\left(\tau_{2}\right)$. This results in the reformulation of the zeroth-order solution

$$
\varepsilon_{n, x}^{(0)}=\varepsilon_{o}\left(\tau_{2}\right) e^{i k n a} e^{-i \omega_{0}^{\prime} \tau_{0}}+\bar{\varepsilon}_{0}\left(\tau_{2}\right) e^{-i k n a} e^{i \omega_{0}^{\prime} \tau_{0}}
$$

as well as the reformulation of the homogeneous solution

$$
\varepsilon_{n, x, h}^{(1)}=\eta_{0}\left(\tau_{2}\right) e^{i k n a} e^{-i \omega_{0}^{\prime} \tau_{0}}+\bar{\eta}_{0}\left(\tau_{2}\right) e^{-i k n a} e^{i \omega_{0}^{\prime} \tau_{0}} .
$$

We finally rewrite Eq. (A15) by calculating the term between the square brackets. After lengthy algebraic manipulations, 
Eq. (A15) becomes

$$
\begin{aligned}
& \frac{\partial \varepsilon_{n, x}^{(1)}}{\partial \tau_{0}}+2 \frac{J}{\hbar} S_{z}^{0}\left(\varepsilon_{n+1, y}^{(1)}-2 \varepsilon_{n, y}^{(1)}+\varepsilon_{n-1, y}^{(1)}\right) \\
& =2 \frac{J}{\hbar} S_{z}^{0} 2(\sin (2 k a)-2 \sin (k a))\left(\varepsilon_{0} A_{0} e^{2 i k n a} e^{-i\left(\omega_{0}+\omega_{0}^{\prime}\right) \tau_{0}}\right. \\
& \left.\quad+\bar{\varepsilon}_{0} \bar{A}_{0} e^{-2 i k n a} e^{i\left(\omega_{0}+\omega_{0}^{\prime}\right) \tau_{0}}\right)
\end{aligned}
$$

We proceed in the same way for the equation of motion in Eq. (A4b). We find that we need to reformulate the zeroth-order solution

$$
\varepsilon_{n, y}^{(0)}=-i \varepsilon_{o}\left(\tau_{2}\right) e^{i k n a} e^{-i \omega_{0}^{\prime} \tau_{0}}+i \bar{\varepsilon}_{0}\left(\tau_{2}\right) e^{-i k n a} e^{i \omega_{0}^{\prime} \tau_{0}}
$$

as well as the homogeneous solution

$$
\varepsilon_{n, y, h}^{(1)}=-i \eta_{0}\left(\tau_{2}\right) e^{i k n a} e^{-i \omega_{0}^{\prime} \tau_{0}}+i \bar{\eta}_{0}\left(\tau_{2}\right) e^{-i k n a} e^{i \omega_{0}^{\prime} \tau_{0}} .
$$
form

The equation of motion in Eq. (A4b) is rewritten in the

$$
\begin{aligned}
& \frac{\partial \varepsilon_{n, y}^{(1)}}{\partial \tau_{0}}-2 \frac{J}{\hbar} S_{z}^{0}\left(\varepsilon_{n+1, x}^{(1)}-2 \varepsilon_{n, x}^{(1)}+\varepsilon_{n-1, x}^{(1)}\right) \\
& =-2 \frac{J}{\hbar} S_{z}^{0} 2 i(\sin (2 k a)-2 \sin (k a))\left(\varepsilon_{0} A_{0} e^{2 i k n a} e^{-i\left(\omega_{0}+\omega_{0}^{\prime}\right) \tau_{0}}\right. \\
& \left.\quad-\bar{\varepsilon}_{0} \bar{A}_{0} e^{-2 i k n a} e^{i\left(\omega_{0}+\omega_{0}^{\prime}\right) \tau_{0}}\right) .
\end{aligned}
$$

Particular solutions of Eqs. (A18) and (A21) can be introduced in the form

$$
\begin{aligned}
& \varepsilon_{n, x, p}^{(1)}=X^{\prime}\left(\tau_{2}\right) e^{2 i k n a} e^{-i\left(\omega_{0}+\omega_{0}^{\prime}\right) \tau_{0}}+\bar{X} \prime\left(\tau_{2}\right) e^{-2 i k n a} e^{i\left(\omega_{0}+\omega_{0}^{\prime}\right) \tau_{0}}, \\
& \varepsilon_{n, y, p}^{(1)}=Y^{\prime}\left(\tau_{2}\right) e^{2 i k n a} e^{-i\left(\omega_{0}+\omega_{0}^{\prime}\right) \tau_{0}}+\overline{Y^{\prime}}\left(\tau_{2}\right) e^{-2 i k n a} e^{i\left(\omega_{0}+\omega_{0}^{\prime}\right) \tau_{0}} .
\end{aligned}
$$

Inserting these particular solutions into Eqs. (A18) and (A21) and solving for $X^{\prime}, Y^{\prime}, \overline{X^{\prime}}, \overline{Y^{\prime}}$ yields

$$
\begin{aligned}
& X^{\prime}=-i \varepsilon_{0} A_{0} f(k), \\
& Y^{\prime}=-i X^{\prime}=-\varepsilon_{0} A_{0} f(k), \\
& \overline{X^{\prime}}=i \bar{\varepsilon}_{0} \bar{A}_{0} f(k), \\
& \overline{Y^{\prime}}=i \overline{X^{\prime}}=-\bar{\varepsilon}_{0} \bar{A}_{0} f(k),
\end{aligned}
$$

where we define $f(k)=\frac{\frac{4}{\hbar} S_{z}^{0}(\sin (2 k a)-2 \sin (k a))}{\frac{8 J}{\hbar} S_{z}^{0} \sin ^{2}(k a)-\left(\omega_{0}+\omega_{0}^{\prime}\right)+i \psi}$. We have introduced a damping term in the definition of $f(k)$ since its denominator may become zero. We will take the limit $\psi \rightarrow 0$ at the end of our derivation.

Adding the homogeneous and particular solutions gives the first-order solutions

$$
\begin{gathered}
\varepsilon_{n, x}^{(1)}=\eta_{0}\left(\tau_{2}\right) e^{i k n a} e^{-i \omega_{0}^{\prime} \tau_{0}}+\bar{\eta}_{0}\left(\tau_{2}\right) e^{-i k n a} e^{i \omega_{0}^{\prime} \tau_{0}}-f(k)\left(i \varepsilon_{0} A_{0} e^{2 i k n a} e^{-i\left(\omega_{0}+\omega_{0}^{\prime}\right) \tau_{0}}-i \bar{\varepsilon}_{0} \bar{A}_{0} e^{-2 i k n a} e^{i\left(\omega_{0}+\omega_{0}^{\prime}\right) \tau_{0}}\right), \\
\varepsilon_{n, y}^{(1)}=-i \eta_{0}\left(\tau_{2}\right) e^{i k n a} e^{-i \omega_{0}^{\prime} \tau_{0}}+i \bar{\eta}_{0}\left(\tau_{2}\right) e^{-i k n a} e^{i \omega_{0}^{\prime} \tau_{0}}-f(k)\left(\varepsilon_{0} A_{0} e^{2 i k n a} e^{-i\left(\omega_{0}+\omega_{0}^{\prime}\right) \tau_{0}}+\bar{\varepsilon}_{0} \bar{A}_{0} e^{-2 i k n a} e^{i\left(\omega_{0}+\omega_{0}^{\prime}\right) \tau_{0}}\right) .
\end{gathered}
$$

We can now address the solutions of the first-order Eq. (A4c). We have to set $\frac{\partial \varepsilon_{n, z}^{(0)}}{\partial \tau_{1}}=0$ (i.e., make $\varepsilon_{n, z}^{(0)}$ independent of $\tau_{1}$ ) to eliminate secular solutions. Inserting the zeroth- and first-order solutions into Eq. (A4) results in

$$
\frac{\partial \varepsilon_{n, z}^{(1)}}{\partial \tau_{0}}=\frac{8 J}{\hbar} f(k) \varepsilon_{0} \bar{\varepsilon}_{0}(\cos (k a)-2 \cos (2 k a))\left(A_{0} e^{i k n a} e^{-i \omega_{0} \tau_{0}}+\bar{A}_{0} e^{-i k n a} e^{i \omega_{0} \tau_{0}}\right) .
$$

Solutions of Eq. (A25) are then easily obtained to within a constant of integration which may be taken equal to zero

$$
\varepsilon_{n, z}^{(1)}=\frac{8 J}{\hbar} f(k) \varepsilon_{0} \bar{\varepsilon}_{0}(\cos (k a)-2 \cos (2 k a))\left(\frac{1}{i \omega_{0}}\right)\left(-A_{0} e^{i k n a} e^{-i \omega_{0} \tau_{0}}+\bar{A}_{0} e^{-i k n a} e^{i \omega_{0} \tau_{0}}\right) .
$$

\section{c. Second order in $K$}

We first deal with the Eq. (A5d) for phonons. The terms with derivatives with respect to $\tau_{1}$ are equal to zero. Inserting the zeroth- and first-order solutions determined previously and after extensive algebraic manipulations we obtain

$$
\frac{\partial^{2} u_{n}^{(2)}}{\partial \tau_{0}^{2}}-\omega_{M}^{2}\left(u_{n+1}^{(2)}-2 u_{n}^{(2)}+u_{n-1}^{(2)}\right)=-2 \frac{\partial^{2} u_{n}^{(0)}}{\partial \tau_{0} \partial \tau_{2}}-\frac{8}{m} f(k) g(k) \varepsilon_{0} \bar{\varepsilon}_{0}\left(A_{0} e^{i k n a} e^{-i \omega_{0} \tau_{0}}+\bar{A}_{0} e^{-i k n a} e^{i \omega_{0} \tau_{0}}\right),
$$

where the function $g(k)$ is given by the expression

$$
g(k)=\frac{4 J}{\hbar} S_{z}^{0} \frac{1}{\omega_{0}}(\cos (k a)-\cos (2 k a)) \sin (k a)+\sin (2 k a)-\sin (k a) .
$$

We subsequently define

$$
A_{0}\left(\tau_{2}\right)=\alpha\left(\tau_{2}\right) e^{-i \varphi\left(\tau_{2}\right)}
$$

and

$$
\bar{A}_{0}\left(\tau_{2}\right)=\alpha\left(\tau_{2}\right) e^{+i \varphi\left(\tau_{2}\right)}
$$


With this definition, the term

$$
\begin{aligned}
-2 \frac{\partial^{2} u_{n}^{(0)}}{\partial \tau_{0} \partial \tau_{2}} & =-2\left(-i \omega_{0} \frac{\partial A_{0}\left(\tau_{2}\right)}{\partial \tau_{2}} e^{i k n a} e^{-i \omega_{0} \tau_{0}}+i \omega_{0} \frac{\partial \bar{A}_{0}\left(\tau_{2}\right)}{\partial \tau_{2}} e^{-i k n a} e^{i \omega_{0} \tau_{0}}\right) \\
& =e^{i k n a} e^{-i \omega_{0} \tau_{0}} 2 \omega_{0} e^{-i \varphi}\left(\frac{\partial \varphi}{\partial \tau_{2}} \alpha+i \frac{\partial \alpha}{\partial \tau_{2}}\right)+e^{-i k n a} e^{i \omega_{0} \tau_{0}} 2 \omega_{0} e^{i \varphi}\left(\frac{\partial \varphi}{\partial \tau_{2}} \alpha-i \frac{\partial \alpha}{\partial \tau_{2}}\right) .
\end{aligned}
$$

The homogeneous equation (i.e., left-hand term of Eq. (A27) set equal to zero) has solutions of the form $e^{i k n a} e^{-i \omega_{0} \tau_{0}}$ and $e^{-i k n a} e^{i \omega_{0} \tau_{0}}$; so to avoid secular terms, the right-hand side of Eq. (A27) must be equal to zero. This gives the two relations

$$
2 \omega_{0} e^{-i \varphi}\left(\frac{\partial \varphi}{\partial \tau_{2}} \alpha+i \frac{\partial \alpha}{\partial \tau_{2}}\right)=\frac{8}{m} f(k) g(k) \varepsilon_{0} \bar{\varepsilon}_{0} \alpha e^{-i \varphi}
$$

and

$$
2 \omega_{0} e^{i \varphi}\left(\frac{\partial \varphi}{\partial \tau_{2}} \alpha-i \frac{\partial \alpha}{\partial \tau_{2}}\right)=\frac{8}{m} f(k) g(k) \varepsilon_{0} \bar{\varepsilon}_{0} \alpha e^{i \varphi} .
$$

Since $\varepsilon_{0} \bar{\varepsilon}_{0}$ is real, one must have $\frac{\partial \alpha}{\partial \tau_{2}}=0$, and $\alpha=\alpha_{0}$ is a constant. Both preceding equations give the same relation

$$
\frac{\partial \varphi}{\partial \tau_{2}}=\frac{4}{m \omega_{0}} f(k) g(k) \varepsilon_{0} \bar{\varepsilon}_{0} .
$$

This equation leads to a linear dependency of $\varphi$ on the time $\tau_{2}$ to within an arbitrary constant (which is taken to be zero)

$$
\varphi=\frac{4}{m \omega_{0}} f(k) g(k) \varepsilon_{0} \bar{\varepsilon}_{0} \tau_{2}=\frac{4}{m \omega_{0}} f(k) g(k) \varepsilon_{0} \bar{\varepsilon}_{0} K^{2} \tau_{0}=K^{2} h(k) \tau_{0} .
$$

This implies that the zeroth-order solution given by Eq. (A13) for the displacement includes a frequency shift. The complete zeroth-order solution for the displacement is now

$$
u_{n}^{(0)}\left(\tau_{0}, \tau_{2}\right)=\alpha_{0} e^{i k n a} e^{-i\left(\omega_{0}+K^{2} h(k)\right) \tau_{0}}+\bar{\alpha}_{0} e^{-i k n a} e^{i\left(\omega_{0}+K^{2} h(k)\right) \tau_{0}} .
$$

The frequency of the phonon is shifted from $\omega_{0}$ to $\omega_{0}+K^{2} h(k)$.

We now address the second-order equations of motion for $\varepsilon_{n, x}^{(2)}$ and for $\varepsilon_{n, y}^{(2)}$; that is, Eqs. (A5a) and (A5b). For this, we need to insert the expressions for the complete zeroth-order displacement [Eq. (A31)] and the first-order displacement given by Eq. (A14). We also need the zeroth-order solutions for $\varepsilon$ [Eqs. (A16) and (A19)] and their first-order expressions given by Eqs. (A24a) and (A24b). Both equations of motion become

$$
\begin{aligned}
\frac{\partial \varepsilon_{n, x}^{(2)}}{\partial \tau_{0}} & +2 \frac{J}{\hbar} S_{z}^{0}\left(\varepsilon_{n+1, y}^{(2)}-2 \varepsilon_{n, y}^{(2)}+\varepsilon_{n-1, y}^{(2)}\right) \\
= & -\frac{\partial \varepsilon_{n, x}^{(0)}}{\partial \tau_{2}}-\frac{4}{\hbar} S_{z}^{0}(\sin (2 k a)-2 \sin (k a))\left[\left(\eta_{0} A_{0}+\varepsilon_{0} B_{0}\right) e^{i 2 k n a} e^{-i\left(\omega_{0}+\omega_{0}^{\prime}\right) \tau_{0}}-\left(\bar{\eta}_{0} \bar{A}_{0}+\bar{\varepsilon}_{0} \bar{B}_{0}\right) e^{-i 2 k n a} e^{i\left(\omega_{0}+\omega_{0}^{\prime}\right) \tau_{0}}\right] \\
& -i \frac{4}{\hbar} S_{z}^{0} f(k)(\sin (3 k a)-\sin (2 k a)-\sin (k a))\left(\varepsilon_{0} A_{0}^{2} e^{i 3 k n a} e^{-i\left(2 \omega_{0}+\omega_{0}^{\prime}\right) \tau_{0}}-\bar{\varepsilon}_{0} \bar{A}_{0}^{2} e^{-i 3 k n a} e^{i\left(2 \omega_{0}+\omega_{0}^{\prime}\right) \tau_{0}}\right) \\
& -i \frac{4}{\hbar} S_{z}^{0} f(k) A_{0} \bar{A}_{0}(\sin (2 k a)-2 \sin (k a))\left(-\varepsilon_{o} e^{i k n a} e^{-i \omega_{0}^{\prime} \tau_{0}}+\bar{\varepsilon}_{0} e^{-i k n a} e^{i \omega_{0}^{\prime} \tau_{0}}\right) \\
\frac{\partial \varepsilon_{n, y}^{(2)}}{\partial \tau_{0}}- & -2 \frac{J}{\hbar} S_{z}^{0}\left(\varepsilon_{n+1, x}^{(2)}-2 \varepsilon_{n, x}^{(2)}+\varepsilon_{n-1, x}^{(2)}\right) \\
= & -\frac{\partial \varepsilon_{n, y}^{(0)}}{\partial \tau_{2}}-i \frac{4}{\hbar} S_{z}^{0}(\sin (2 k a)-2 \sin (k a))\left[\left(\eta_{0} A_{0}+\varepsilon_{0} B_{0}\right) e^{i 2 k n a} e^{-i\left(\omega_{0}+\omega_{0}^{\prime}\right) \tau_{0}}-\left(\bar{\eta}_{0} \bar{A}_{0}+\bar{\varepsilon}_{0} \bar{B}_{0}\right) e^{-i 2 k n a} e^{i\left(\omega_{0}+\omega_{0}^{\prime}\right) \tau_{0}}\right] \\
\quad & -\frac{4}{\hbar} S_{z}^{0} f(k)(\sin (3 k a)-\sin (2 k a)-\sin (k a))\left(\varepsilon_{0} A_{0}^{2} e^{i 3 k n a} e^{-i\left(2 \omega_{0}+\omega_{0}^{\prime}\right) \tau_{0}}+\bar{\varepsilon}_{0} \bar{A}_{0}^{2} e^{-i 3 k n a} e^{i\left(2 \omega_{0}+\omega_{0}^{\prime}\right) \tau_{0}}\right) \\
& +\frac{4}{\hbar} S_{z}^{0} f(k) A_{0} \bar{A}_{0}(\sin (2 k a)-2 \sin (k a))\left(\varepsilon_{o} e^{i k n a} e^{-i \omega_{0}^{\prime} \tau_{0}}+\bar{\varepsilon}_{0} e^{-i k n a} e^{i \omega_{0}^{\prime} \tau_{0}}\right)
\end{aligned}
$$


Only the terms in $e^{i k n a} e^{-i \omega_{0}^{\prime} \tau_{0}}$ and $e^{-i k n a} e^{i \omega_{0}^{\prime} \tau_{0}}$ will give secular solutions. To eliminate these solutions, we set

$$
\begin{aligned}
& 0=-\frac{\partial \varepsilon_{n, x}^{(0)}}{\partial \tau_{2}}-i \frac{4}{\hbar} S_{z}^{0} f(k) A_{0} \bar{A}_{0}(\sin (2 k a)-2 \sin (k a))\left(-\varepsilon_{o} e^{i k n a} e^{-i \omega_{0}^{\prime} \tau_{0}}+\bar{\varepsilon}_{0} e^{-i k n a} e^{i \omega_{0}^{\prime} \tau_{0}}\right), \\
& 0=-\frac{\partial \varepsilon_{n, y}^{(0)}}{\partial \tau_{2}}+\frac{4}{\hbar} S_{z}^{0} f(k) A_{0} \bar{A}_{0}(\sin (2 k a)-2 \sin (k a))\left(\varepsilon_{o} e^{i k n a} e^{-i \omega_{0}^{\prime} \tau_{0}}+\bar{\varepsilon}_{0} e^{-i k n a} e^{i \omega_{0}^{\prime} \tau_{0}}\right) .
\end{aligned}
$$

Using the expressions previously derived for $\varepsilon_{n, x}^{(0)}$ and $\varepsilon_{n, y}^{(0)}$, these two conditions reduce to

$$
\begin{aligned}
& \frac{\partial \varepsilon_{0}}{\partial \tau_{2}}=i \frac{4}{\hbar} S_{z}^{0} f(k) A_{0} \bar{A}_{0}(\sin (2 k a)-2 \sin (k a)) \varepsilon_{0}\left(\tau_{2}\right), \\
& \frac{\partial \bar{\varepsilon}_{0}}{\partial \tau_{2}}=-i \frac{4}{\hbar} S_{z}^{0} f(k) A_{0} \bar{A}_{0}(\sin (2 k a)-2 \sin (k a)) \bar{\varepsilon}_{0}\left(\tau_{2}\right) .
\end{aligned}
$$

Similarly to finding the second-order solution for the displacement, we define $\varepsilon_{0}\left(\tau_{2}\right)=\lambda\left(\tau_{2}\right) e^{-i \varphi^{\prime}\left(\tau_{2}\right)}$ and $\bar{\varepsilon}_{0}\left(\tau_{2}\right)=\lambda\left(\tau_{2}\right) e^{i \varphi \prime\left(\tau_{2}\right)}$. Inserting these expressions into the preceding conditions leads to taking $\lambda\left(\tau_{2}\right)=\lambda_{0}$ (i.e., a constant) and

$$
\varphi^{\prime}\left(\tau_{2}\right)=-\frac{4}{\hbar} S_{z}^{0} f(k) \alpha_{0}^{2}(\sin (2 k a)-2 \sin (k a)) \tau_{2}=K^{2} h^{\prime}(k) \tau_{0} .
$$

To arrive at that equation, we have assumed that the constant of integration is zero.

Equation (A33) introduces a correction to the zeroth-order solutions for $\varepsilon_{n, x}^{(0)}$ and $\varepsilon_{n, y}^{(0)}$ which become

$$
\begin{gathered}
\varepsilon_{n, x}^{(0)}=\lambda_{o} e^{i k n a} e^{-i\left(\omega_{0}^{\prime}+K^{2} h^{\prime}(k)\right) \tau_{0}}+\bar{\lambda}_{0} e^{-i k n a} e^{i\left(\omega_{0}^{\prime}+K^{2} h^{\prime}(k)\right) \tau_{0}}, \\
\varepsilon_{n, y}^{(0)}=-i \lambda_{o} e^{i k n a} e^{-i\left(\omega_{0}^{\prime}+K^{2} h^{\prime}(k)\right) \tau_{0}}+i \bar{\lambda}_{0}\left(\tau_{2}\right) e^{-i k n a} e^{i\left(\omega_{0}^{\prime}+K^{2} h^{\prime}(k)\right) \tau_{0}} .
\end{gathered}
$$

Similarly to the phonons, the magnons are frequency shifted.

The denominator of the function $f(k)$ was written as

$$
d(k)=8 \frac{J}{\hbar} S_{z}^{0} \sin ^{2}(k a)-\left(\omega_{0}+\omega_{0}^{\prime}\right)+i \psi
$$

The introduction of the small damping $i \psi$ enables us to overcome the divergence at the resonance. Indeed, we can take the following limit using Sokhotski’s formula [32]

$$
\begin{aligned}
\lim _{\psi \rightarrow 0} \frac{1}{d(k)} & =\lim _{\psi \rightarrow 0} \frac{1}{8 \frac{J}{\hbar} S_{z}^{0} \sin ^{2}(k a)-\left(\omega_{0}(k)+\omega_{0}^{\prime}(k)\right)+i \psi} \\
& =\left[\frac{1}{8 \frac{J}{\hbar} S_{z}^{0} \sin ^{2}(k a)-\left(\omega_{0}(k)+\omega_{0}^{\prime}(k)\right)}\right]_{p v}-i \pi \delta\left(8 \frac{J}{\hbar} S_{z}^{0} \sin ^{2}(k a)-\left(\omega_{0}(k)+\omega_{0}^{\prime}(k)\right) .\right.
\end{aligned}
$$

In the previous equation, the bracket []$_{p v}$ means Cauchy's principal value.

The shift in frequency of the magnons and phonons becomes finite with a damping at the resonance. The imaginary term with the delta function leads to a finite lifetime for the phonon and magnon excitations at the resonance.

[1] M. Johnson and R. H. Silsbee, Phys. Rev. Lett. 55, 1790 (1985).

[2] F. J. Jedema, A. T. Filip, and B. J. van Wees, Nature 410, 345 (2001).

[3] Y. Tserkovnyak, A. Brataas, and G. Bauer, Phys. Rev. Lett. 88, 117601 (2002).

[4] A. Brataas, Y. Tserkovnyak, G. E. W. Bauer, and B. I. Halperin, Phys. Rev. B 66, 060404(R) (2002).

[5] S. Mizukami, Y. Ando, and T. Miyazaki, Phys. Rev. B 66, 104413 (2002).

[6] T. Jungwirth, J. Wunderlich, and K. Olejnik, Nat. Mater. 11, 382 (2012).

[7] A. Hoffman, IEEE Trans. Magn. 49, 5172 (2013).

[8] K. Uchida, S. Takahashi, K. Harii, J. Ieda, W. Koshibae, K. Ando, S. Maekawa, and E. Saitoh, Nature 455, 778 (2008).

[9] K. Uchida, Nat. Mater. 9, 894 (2010).
[10] Y. Kajiwara, K. Harii, S. Takahashi, J. Ohe, K. Uchida, M. Mizuguchi, H. Umezawa, H. Kawai, K. Ando, K. Takanashi, S. Maekawa, and E. Saitoh, Nature (London) 464, 262 (2010).

[11] A. V. Scherbakov, A. S. Salasyuk, A. V. Akimov, X. Liu, M. Bombeck, C. Brüggemann, D. R. Yakovlev, V. F. Sapega, J. K. Furdyna, and M. Bayer, Phys. Rev. Lett. 105, 117204 (2010).

[12] J.-W. Kim, M. Vomir, and J.-Y. Bigot, Phys. Rev. Lett. 109, 166601 (2012).

[13] M. Weiler, H. Huebl, F. S. Goerg, F. D. Czeschka, R. Gross, and S. T. B. Goennenwein, Phys. Rev. Lett. 108, 176601 (2012).

[14] K. Uchida, H. Adachi, T. An, T. Ota, M. Toda, B. Hillebrands, S. Maekawa, and E. Saitoh, Nat. Mater. 10, 737 (2011). 
[15] M. Weiler, L. Dreher, C. Heeg, H. Huebl, R. Gross, M. S. Brandt, and S. T. B. Goennenwein, Phys. Rev. Lett. 106, 117601 (2011).

[16] H. Adachi, K. Uchida, E. Saitoh, J. Ohe, S. Takahashi, and S. Maekawa, Appl. Phys. Lett. 97, 252506 (2010).

[17] T. An, V. I. Vasyuchka, K. Uchida, A. V. Chumak, K. Yamaguchi, K. Harii, J. Ohe, M. B. Jungfleisch, Y. Kajiwara, H. Adachi, B. Hillebrands, S. Maekawa, and E. Saitoh, Nat. Mater. 12, 549 (2013).

[18] G. W. Bauer, E. Saitoh, and B. J. van Wees, Nat. Mater. 11, 391 (2012),

[19] P. A Fedders, Phys. Rev. B 9, 3835 (1974).

[20] T. Kobayashi, R. C. Barker, J. L. Bleustein, and A. Yelon, Phys. Rev. B 7, 3273 (1973).

[21] H. F. Tiersten, J. Math. Phys. 5, 1298 (1964).

[22] D. Perera, D. P. Landau, D. M. Nicholson, G. M. Stocks, M. Eisenbach, J. Yin, and G. Brown, J. Appl. Phys. 115, 17D124 (2014).
[23] P.-W. Ma, S. L. Dudarev, and C. H. Woo, J. Appl. Phys. 111, 07D114 (2012).

[24] P.-W. Ma, C. H. Woo, and S. L. Dudarev, Phys. Rev. B 78, 024434 (2008).

[25] I. C. Khoo and Y. K. Wang, J. Math. Phys. 17, 222 (1976).

[26] O. Bou Matar, J. F. Robillard, J. O. Vasseur, A.-C. HladkyHennion, P. A. Deymier, P. Pernod, and V. Preobrazhensky, J. Appl. Phys. 111, 054901 (2012).

[27] R. Skomski, Simple Models of Magnetism (Oxford University Press, Oxford, 2008).

[28] R. Silberglitt, Phys. Rev. 188, 786 (1969).

[29] L. M. Woods, Phys. Rev. B 65, 014409 (2001).

[30] J. O. Vasseur, L. Dobrzynski, B. Djafari-Rouhani, and H. Puszkarski, Phys. Rev. B 54, 1043 (1996).

[31] Acoustic Metamaterials and Phononic Crystals, Springer Series in Solid-State Sciences Vol. 173, edited by P. A. Deymier (Springer, Berlin, 2013).

[32] V. S. Vladimirov, Equations of Mathematical Physics (Marcel Dekker, New York, 1971). 